TRANSACTIONS OF THE

AMERICAN MATHEMATICAL SOCIETY

Volume 353, Number 3, Pages 943-962

S 0002-9947(00)02717-3

Article electronically published on October 11, 2000

\title{
MEASURING THE TAMENESS OF ALMOST CONVEX GROUPS
}

\author{
SUSAN HERMILLER AND JOHN MEIER
}

\begin{abstract}
A 1-combing for a finitely presented group consists of a continuous family of paths based at the identity and ending at points $x$ in the 1-skeleton of the Cayley 2-complex associated to the presentation. We define two functions (radial and ball tameness functions) that measure how efficiently a 1-combing moves away from the identity. These functions are geometric in the sense that they are quasi-isometry invariants. We show that a group is almost convex if and only if the radial tameness function is bounded by the identity function; hence almost convex groups, as well as certain generalizations of almost convex groups, are contained in the quasi-isometry class of groups admitting linear radial tameness functions.
\end{abstract}

\section{INTRODUCTION}

In the late 80 s and early 90 s, various ideas were imported from the study of 3 -manifolds to the study of finitely presented infinite groups; in particular, ideas related to the covering conjecture and missing boundary problems were found to be useful. Highlights include Cannon's almost convexity and the notion of tame 1-combings due to Mihalik and Tschantz. The tame 1-combing property has one notable advantage over almost convexity: admitting a tame 1-combing is a geometric property in the sense that it does not depend on choice of presentation [13, while almost convexity does depend on presentation 18 .

In an earlier paper [6] we showed how the ideas of almost convexity, tame combings, and the more algorithmic idea of rewriting systems, interrelate. Looking over that work we realized that the tame combings arising from almost convexity were very tame. Our primary goal in this paper is to introduce a method of measuring the "tameness" of a given tame 1-combing, and to show that this degree of tameness - much like isodiametric and isoperimetric functions - is a quasi-isometry invariant.

In order to state our main theorems, we quickly review the definitions of tame combing and almost convexity. Let $\widetilde{X}$ be the Cayley 2-complex for a finitely presented group $G$, and pick a basepoint $\epsilon \in \widetilde{X}^{0}$. More generally, $\widetilde{X}$ can be the universal cover of a finite, connected, 2-dimensional PLCW-complex $X$. A 0 -combing of $\widetilde{X}$ is a collection of paths $\Psi: \widetilde{X}^{0} \times[0,1] \rightarrow \widetilde{X}^{1}$, where $\left.\Psi\right|_{v \times[0,1]}$ begins at $\epsilon$ and ends at $v \in \widetilde{X}^{0}$. Viewing $\widetilde{X}^{1}$ as the Cayley graph of the group $G$, this can

Received by the editors June 21, 1999.

2000 Mathematics Subject Classification. Primary 20F65; Secondary 20F69, 57M07.

Key words and phrases. Tame combings, almost convex groups, covering conjecture, rewriting systems, isoperimetric inequalities.

Susan Hermiller acknowledges support from NSF grant DMS-9623088.

John Meier acknowledges support from NSF RUI grant DMS-9704417.

(C)2000 American Mathematical Society 
be thought of as the standard notion of a combing for $G$. The map $\Psi$ is a tame 0-combing if for each compact set $C \subseteq \widetilde{X}$ there is a compact set $D \subseteq \widetilde{X}$ such that for all $x \in \widetilde{X}^{0}, \Psi^{-1}(C) \cap(\{x\} \times[0,1])$ is contained in a single path component of $\Psi^{-1}(D) \cap(\{x\} \times[0,1])$.

Similarly, a 1-combing of $\widetilde{X}$ is a homotopy $\Psi: \widetilde{X}^{1} \times[0,1] \rightarrow \widetilde{X}$ such that $\Psi(x, 0)=\epsilon$ and $\Psi(x, 1)=x$ for all $x \in \widetilde{X}^{1}$, with the condition that the restriction of $\Psi$ to $\widetilde{X}^{0} \times[0,1]$ is a 0 -combing. The map $\Psi$ is a tame 1 -combing if this restriction is a tame 0-combing, and if for each compact set $C \subseteq \widetilde{X}$ there is a compact set $D \subseteq \widetilde{X}$ such that for all edges $e \subseteq \widetilde{X}^{1}, \Psi^{-1}(C) \cap(e \times[0,1])$ is contained in a single path component of $\Psi^{-1}(D) \cap(e \times[0,1])$. This is often expressed as: once a combing path leaves $D$ it never returns to $C$.

Cannon's almost convexity condition can be defined in terms of the metric structure of Cayley graphs. Let $\widetilde{X}^{1}$ be the Cayley graph for a finitely generated group, with the path metric. If $n$ is any positive integer, then the sphere of radius $n$ is $S(n)=\left\{x \in \widetilde{X}^{1} \mid d(\epsilon, x)=n\right\}$ where $\epsilon$ is the identity element; similarly, the ball of radius $n$ is $B(n)=\left\{x \in \widetilde{X}^{1} \mid d(\epsilon, x) \leq n\right\}$.

A group $G$ is almost convex 2 ] with respect to a finite symmetric set of generators if there is a constant $A$ such that for any integer $n$ and any pair of group elements $g, h \in G$ with $g, h \in S(n)$ and $d(g, h) \leq 2$, there is a path in $B(n)$ of length at most $A$ joining $g$ and $h$. A short exercise shows that the existence of $A$ implies there is a list of constants $\left\{A_{k}\right\}$ (one for each integer $k \geq 2$ ) such that points in $S(n)$ that are a distance $\leq k$ apart can be joined in $B(n)$ by a path of length at most $A_{k}$.

We measure the "tameness" of a tame 1-combing in two ways. First, a tame 1-combing $\Psi$ admits a radial tameness function $\rho: \mathbf{Q} \rightarrow \mathbf{Q}$ if for any point $x$ in $\widetilde{X}^{1}, s \in[0,1]$, and $q \in \mathbf{Q}$, whenever $\Psi(x, s)$ leaves $B(\rho(q))$, then all of the points $\Psi(x, t)$ with $t>s$ are outside $B(q)$. (We describe what we mean by a "ball of radius $q "$ in a Cayley complex as opposed to the Cayley graph in Section 2; this is accomplished through the introduction of levels.) Note that $\rho(q) \geq q$ for all $q$.

Theorem A. Suppose that $X$ and $Y$ are finite, connected 2-dimensional PLCWcomplexes whose fundamental groups are quasi-isometric. Let $\widetilde{X}$ and $\widetilde{Y}$ be their corresponding universal covers. Then $\tilde{X}$ has a tame 1-combing that admits a radial tameness function $\rho_{X}$ if and only if $\widetilde{Y}$ has a tame 1-combing that admits an equivalent radial tameness function $\rho_{Y}$.

Theorem C. A finitely presented group $G$ is almost convex with respect to a finite set of generators if and only if there is a finite presentation, with the same set of generators, such that the associated Cayley complex admits a tame 1-combing with radial tameness function $\rho(q)=q$.

Combining Theorems $\mathrm{A}$ and $\mathrm{C}$ we see that the collection of almost convex groups is contained in the quasi-isometry class of groups that admit a linear radial tameness function. This result is generalized in Theorem D to groups satisfying weaker versions of almost convexity.

While the class of groups admitting linear radial tameness functions may be large, it certainly does not include all finitely presented groups. For example, in $\S 5$ we prove there are groups whose radial tameness functions are at least multiply exponential.

In $\S 6$ we explore a second notion of tameness that, in some sense, measures the width rather than the length of a tame combing. Let $e$ be any edge of $\tilde{X}$. We 
have restricted ourselves to PLCW complexes, so by simplicial approximation, we may assume that the maps $\left.\Psi\right|_{e \times[0,1]}$ are combinatorial, that is, the domain $e \times[0,1]$ can be subdivided so that $\left.\Psi\right|_{e \times[0,1]}$ maps cells isomorphically to cells. The tame combing $\Psi$ admits a ball tameness function $\beta: \mathbf{Q} \rightarrow \mathbf{Q}$ if for any edge $e$ and $q \in \mathbf{Q}$, the number of vertices, edges, and 2-cells in $e \times[0,1]$ which map to cells in $B(q)$ is bounded by $\beta(q)$. That is, we are counting the number of cells in $B(q)$ that are hit by $\Psi$, with multiplicity. Note that a tame combing $\Psi$ may have a value of $\beta(q)$ that is not finite; in this case, we say the combing does not admit a ball tameness function.

Like radial tameness, the ball tameness function is a quasi-isometry invariant (Theorem B). Combining radial and ball tameness functions allows us to bound the isoperimetric and isodiametric functions of $G$ (Theorem E). In particular, as a corollary to these results we show: If a group $G$ has a tame combing admitting recursive radial and ball tameness functions, then $G$ has recursive isoperimetric and isodiametric functions, and the word problem for $G$ is solvable.

\section{Definitions}

\subsection{Geometry of the Cayley complex.}

Suppose $G$ is a finitely presented group. We assume that for every presentation in this paper, the set of generators is closed under inverses, or symmetric, none of the generators has order 1 , and no two generators are equal in the group. Let $\widetilde{X}$ be the universal cover of the standard 2-complex, or Cayley 2-complex, associated to the finite presentation. Let $\widetilde{X}^{0}$ denote the 0-skeleton; the vertices in $\widetilde{X}^{0}$ are in one-to-one correspondence with the elements of the group. Also, let $\widetilde{X}^{1}$ denote the 1-skeleton of $\widetilde{X}$, i.e. the Cayley graph.

As a reminder, because it is sometimes convenient to stray from presentation 2-complexes to more general finite 2-complexes, we allow $\widetilde{X}$ to be the universal cover of any finite, connected, PLCW-2-complex X. (See $\S I .1 .4$ of [7] for definitions and background on PLCW complexes.) We will abuse notation, and continue to call $\widetilde{X}^{1}$ and $\widetilde{X}$ the Cayley graph and Cayley complex of $\pi_{1}(X)$.

There is a natural metric, called the path metric, on the Cayley graph $\widetilde{X}^{1}$ associated to a finite presentation: each edge is given the metric structure of the unit interval, and the distance between any two points of $\widetilde{X}^{1}$ is the length of the shortest path between the two points. Regrettably, for arbitrary finitely presented groups, there is no simple way to extend this metric to a metric structure on the entire Cayley complex, and this motivates our use of "levels" for the cells of $\widetilde{X}$.

Choose a base vertex $\epsilon \in \widetilde{X}^{0}$. The level of $v$ for a vertex $v$ in $\widetilde{X}^{0}$, denoted $\operatorname{lev}(v)$, is the distance, using the path metric on the 1-skeleton, between the vertex $v$ and the vertex $\epsilon$. For each edge $e$, the level of $e$, denoted lev(e), is $\frac{1}{4}$ plus the average of the levels of the endpoints of $e$. The level of any point of the interior of $e$ is defined to be the same as the level of $e$. Note that for any point $x \in X^{1}$, $d(\epsilon, x)-\frac{1}{4} \leq \operatorname{lev}(x)<d(\epsilon, x)+\frac{3}{4}$, where $d$ denotes the path metric. Finally, for a 2-cell $f$, the level of $f$, written $\operatorname{lev}(f)$, is defined to be the average of the levels of all of its boundary edges, plus $\frac{1}{c}$, where $c=4 n_{1} \cdots n_{k}+1$ and $n_{1}, \ldots, n_{k}$ are the numbers of edges in the polygons used in constructing $X$. The actual value of $c$ is unimportant; what is important is to choose $c$ so that no 2-cell has the same level as a 1- or 0 -cell. 
Recall that a function $f: \mathbf{N} \rightarrow \mathbf{N}$ is an isoperimetric function for $\pi_{1}(X)$ if for any freely reduced word $w \subset \widetilde{X}^{1}$, where $w$ represents the trivial element, there is a van Kampen diagram $D$ with boundary label $w$ containing at most $f(l(w))$ 2-cells. (Here $l(w)$ is the word length of $w$.) A function $g: \mathbf{N} \rightarrow \mathbf{N}$ is an isodiametric function for $\pi_{1}(X)$ if for any freely reduced word $w \subset \widetilde{X}^{1}$ as above, there is a van Kampen diagram $D$ for $w$ such that the (word length) distance from any vertex of $D$ to the basepoint is at most $g(l(w))$. See 9 for information on van Kampen diagrams and [5] for background on the isodiametric and isoperimetric inequalities.

\subsection{Tame combings.}

Tame 1-combings were defined in the introduction. It is shown in [13 that tame combability is equivalent to a property first introduced by Tucker in the context of 3-manifolds $[19$. Let $\widetilde{X}$ be the universal cover of a finite 2-dimensional polyhedron $X$. We say that $\widetilde{X}$ is Tucker if for any finite subcomplex $C \subset \widetilde{X}, \pi_{1}(\widetilde{X}-C)$ is finitely generated (i.e. each path component of $\widetilde{X}-C$ has finitely generated fundamental group).

The equivalence of the Tucker condition and tame combability uses the assumption that $X$ is polyhedral; it is false in the setting of arbitrary CW complexes. Our assumption that all complexes are PLCW also allows us to count the number of times $\left.\Psi\right|_{e \times[0,1]}$ hits cells in $B(q)$, that is, it allows us to define the ball tameness function.

The definitions of radial and ball tameness functions given in the introduction refer to "balls of radius $n$ " in $\widetilde{X}$, although this concept was really only defined for graphs, not 2-complexes. For $q \in \mathbf{Q}$ we define the ball $B(q)$ of radius $q$ to consist of $\{\sigma \in \widetilde{X} \mid \operatorname{lev}(\sigma) \leq q\}$, and note that the usual ball of radius $n$ in the Cayley graph is the intersection of the full Cayley graph with the ball of radius $n$ in the Cayley 2-complex, for any $n \in \mathbf{N}$.

The radial tameness function has an immediate application to the Tucker property. An examination of the Mihalik-Tschantz proof of "tame combable $\Leftrightarrow$ Tucker" establishes the following result.

Proposition 2.1. Let $\widetilde{X}$ be the universal cover of a finite 2-dimensional polyhedron $X$, where $\widetilde{X}$ is tame 1-combable with radial tameness function $\rho$. Then there is a constant $K$ such that, given any finite subcomplex $C \subset \tilde{X}$, if $C \subset B(n)$, then generators for the fundamental group of any component of $\widetilde{X}-C$ can be found in $B(\rho(\rho(n+K)+K))-C$.

We note that Mihalik has also introduced "tame pairs of groups" which, combined with Tucker's results, allow him to get concrete results related to the missing boundary property for 3-manifolds (see [11] and [12]). We hope to consider degrees of tameness for pairs of groups in a later paper.

\subsection{Quasi-isometry.}

Two finitely presented groups $G$ and $H$ are quasi-isometric if there are functions $f: G \rightarrow H$ and $g: H \rightarrow G$ and a constant $k>1$ such that for all $x, w \in G$ and for all $y, z \in H$,

1. $d_{H}(f(x), f(w)) \leq k d_{G}(x, w)+k$,

2. $d_{G}(g(y), g(z)) \leq k d_{H}(y, z)+k$,

3. $d_{G}(g(f(x)), x) \leq k$, and

4. $d_{H}(f(g(y)), y) \leq k$, 
where $d_{G}$ and $d_{H}$ denote the word metrics in $G$ and $H$ respectively. While there is nothing in the definition that requires it, we will be assuming that our quasiisometries take identity elements to identity elements. If $f$ is a quasi-isometry where $f\left(e_{G}\right) \neq e_{H}$, then the map $\widehat{f}: G \rightarrow H$ defined by $\widehat{f}\left(e_{G}\right)=e_{H}$, and $\widehat{f}(x)=f(x)$ otherwise, is a quasi-isometry, although the constant $k$ might need to be larger.

Lemma 2.2. Let $X$ and $Y$ be two finite, connected, $P L C W$-2-complexes whose fundamental groups are quasi-isometric. Then there are cellular maps $\widetilde{f}: \widetilde{X} \rightarrow \widetilde{Y}$ and $\widetilde{g}: \widetilde{Y} \rightarrow \widetilde{X}$, and a constant $K>1$ such that for all $x, w \in \widetilde{X}^{1}$ and for all $y, z \in \tilde{Y}^{1}$,

1. $d_{Y}(\widetilde{f}(x), \widetilde{f}(w)) \leq K d_{X}(x, w)+K$,

2. $d_{X}(\widetilde{g}(y), \widetilde{g}(z)) \leq K d_{Y}(y, z)+K$,

3. $d_{X}(\widetilde{g}(\widetilde{f}(x)), x) \leq K$, and

4. $d_{Y}(\widetilde{f}(\widetilde{g}(y)), y) \leq K$,

where $d_{X}$ and $d_{Y}$ denote the path metrics in $\widetilde{X}^{1}$ and $\widetilde{Y}^{1}$ respectively. Moreover, $\tilde{f}$ and $\widetilde{g}$ can be defined so that for any cell $\sigma$ in $\widetilde{X}$ and $\tau$ in $\widetilde{Y}, \widetilde{f}(\sigma)$ and $\widetilde{g}(\tau)$ contain at most $K$ cells, including cells in all dimensions, and counted with multiplicity. If $\epsilon_{X}$ and $\epsilon_{Y}$ are basepoints in $\widetilde{X}$ and $\widetilde{Y}$, respectively, then $\widetilde{f}$ and $\widetilde{g}$ can also be defined so that $\widetilde{f}\left(\epsilon_{X}\right)=\epsilon_{Y}$ and $\widetilde{g}\left(\epsilon_{Y}\right)=\epsilon_{X}$.

Proof. Let $f$ be the quasi-isometry from the group $G=\pi_{1}(X)$ to $H=\pi_{1}(Y)$, and let $g$ be the complementary quasi-isometry from $\pi_{1}(Y)$ back to $\pi_{1}(X)$, with associated constant $k$. These functions can be thought of as maps between selected vertices in $\widetilde{X}$ and $\widetilde{Y}$. More specifically, let $\epsilon_{X}$ and $\epsilon_{Y}$ denote chosen base vertices in $\widetilde{X}$ and $\tilde{Y}$. Then $f$ and $g$ can be thought of as maps between the $\pi_{1}(X)$ translates of $\epsilon_{X}$ and the $\pi_{1}(Y)$ translates of $\epsilon_{Y}$.

We construct our cellular maps $\tilde{f}$ and $\widetilde{g}$ one dimension at a time. First, on the translates of the basepoints, define $\widetilde{f}_{0}\left(w \cdot \epsilon_{X}\right)=f(w) \cdot \epsilon_{Y}$ for $w \in \pi_{1}(X)$ and $\widetilde{g}_{0}\left(z \cdot \epsilon_{Y}\right)=g(z) \cdot \epsilon_{X}$ for $z \in \pi_{1}(Y)$. Because of our assumption that $f$ and $g$ map identity elements to identity elements, the maps $\widetilde{f}_{0}$ and $\widetilde{g}_{0}$ map basepoints to basepoints. Let $\widetilde{f}_{0}: \widetilde{X}^{0} \rightarrow \widetilde{Y}^{0}$ via $\widetilde{f}_{0}(v)=\widetilde{f}_{0}\left(g_{v} \cdot \epsilon_{X}\right)$, where $g_{v} \cdot \epsilon_{X}$ is a $\pi_{1}(X)$ translate of $\epsilon_{X}$ that is of minimum distance (path metric) from $v$. Since $X$ is a finite complex, the distance from $v$ to $g_{v} \cdot \epsilon_{X}$ will be bounded by the finite number $\left|X^{0}\right|$. Further, one can choose loops in $X$ and $Y$ representing the generators of $G$ and $H$ respectively; let $L$ represent the combinatorial length of the longest such loop. It follows that for any $x, w \in \widetilde{X}^{0}$,

$$
\begin{aligned}
d_{Y}\left(\widetilde{f}_{0}(x), \widetilde{f}_{0}(w)\right) & =d_{Y}\left(\widetilde{f}_{0}\left(g_{x} \cdot \epsilon_{X}\right), \widetilde{f}_{0}\left(g_{w} \cdot \epsilon_{X}\right)\right)=d_{Y}\left(f\left(g_{x}\right) \cdot \epsilon_{Y}, f\left(g_{w}\right) \cdot \epsilon_{Y}\right) \\
& \leq L d_{H}\left(f\left(g_{x}\right), f\left(g_{w}\right)\right) \\
& \leq L\left(k d_{G}\left(g_{x}, g_{w}\right)+k\right) \\
& \leq L\left(k d_{X}\left(g_{x} \cdot \epsilon_{X}, g_{w} \cdot \epsilon_{X}\right)+k\right) \\
& \leq L k\left(d_{X}(x, w)+2\left|X^{0}\right|+1\right)=L k d_{X}(x, w)+2\left|X^{0}\right| L k+L k,
\end{aligned}
$$

so we must pick $K \geq 2\left|X^{0}\right| L k+L k$. Define $\widetilde{g}_{0}: \widetilde{Y}^{0} \rightarrow \widetilde{X}^{0}$ by $\widetilde{g}_{0}(v)=\widetilde{g}_{0}\left(h_{v} \cdot \epsilon_{Y}\right)$ where, again, $h_{v} \cdot \epsilon_{Y}$ is a $\pi_{1}(Y)$ translate of $\epsilon_{Y}$ of minimum distance (at most $\left|Y^{0}\right|$ ) from $v$. The fact that the maps $\widetilde{f}_{0}$ and $\widetilde{g}_{0}$ between the entire 0-skeleta of $\widetilde{X}$ and $\widetilde{Y}$ satisfy (i)-(iv) then follows from the fact that $f$ and $g$ are quasi-isometries. 
We extend the maps $\widetilde{f}_{0}$ and $\widetilde{g}_{0}$ to maps on the 1-skeleta. If $e$ is an edge in $\widetilde{X}$ that is bounded by the vertices $a$ and $b$, define $\tilde{f}_{1}(e)$ to be a minimal length path in $\widetilde{Y}$ connecting $\widetilde{f}_{0}(a)$ to $\widetilde{f}_{0}(b)$. Since we know that

$$
\begin{aligned}
d_{Y}\left(\tilde{f}_{1}(a), \tilde{f}_{1}(b)\right) & =d_{Y}\left(\widetilde{f}_{0}(a), \widetilde{f}_{0}(b)\right) \\
& \leq L k d_{X}(a, b)+2\left|X^{0}\right| L k+L k=2\left|X^{0}\right| L k+2 L k,
\end{aligned}
$$

this number bounds the length of the path $\widetilde{f}_{1}(e)$ for all edges $e$. The map $\widetilde{g}_{1}: \widetilde{Y}^{1} \rightarrow$ $\widetilde{X}^{1}$ is defined similarly.

The maps $\widetilde{f}$ and $\widetilde{g}$ are defined by extending $\widetilde{f}_{1}$ and $\widetilde{g}_{1}$ to the 2 -cells. Since $X$ is a finite complex, there is a constant $J$ such that for any 2-cell $\sigma$ in $\widetilde{X}, \partial \sigma$ is a circuit of at most $J$ edges in $\widetilde{X}^{1}$. Hence $\widetilde{f}_{1}(\partial \sigma)$ is a circuit of at most $J \cdot\left(2\left|X^{0}\right| L k+2 L k\right)$ edges in $\widetilde{Y}^{1}$. Since $\widetilde{Y}$ is simply connected, there is a combinatorial disk, and a cellular map, filling in the circuit $\tilde{f}_{1}(\partial \sigma)$; let $\tilde{f}$ map $\sigma$ to this filling disk. Since there is a bound on the number of edges in this loop, we can also choose $\sigma$ so that we bound the number of cells in $\widetilde{f}(\sigma)$, counted with multiplicity, in all dimensions. (For example, if $\tilde{f}$ mapped a 2-simplex $\sigma$ isomorphically to another 2-simplex, then $\widetilde{f}(\sigma)$ would contain seven cells.) Again, $\widetilde{g}$ is defined similarly.

\section{QUASI-ISOMETRY INVARIANCE}

In 13 Mihalik and Tschantz proved that having a tame combing is independent of presentation. Moreover, if $X$ and $Y$ are two finite PLCW-complexes with $\pi_{1}(X) \simeq \pi_{1}(Y)$, then $\widetilde{X}$ has a tame 1-combing if and only if $\widetilde{Y}$ has a tame 1 combing. Using an alternate definition (the Tucker property mentioned in $\$ 2.2$ ) Brick extended this result, proving that admitting a tame 1-combing is a quasiisometry invariant [1]. However, in the proofs of Theorems A and B we need to make specific reference to the construction of the tame 1-combing for $\tilde{Y}$. Hence we have chosen to include an outline of how the Mihalik-Tschantz proof can be adapted to the quasi-isometry setting. Later in this section we use this result to show that our measures of tameness are quasi-isometry invariants.

Proposition 3.1. Let $X$ and $Y$ be two finite, connected, PLCW-2-complexes whose fundamental groups are quasi-isometric. If $\widetilde{X}$ admits a tame 1-combing, then $\tilde{Y}$ also admits a tame 1-combing.

Both here and elsewhere in the paper we will refer to stars and iterated stars. If $C$ is any subcomplex of $X$, the star of $C$ in $X$, denoted $S t(C)$, is the collection of all cells whose closure has non-trivial intersection with $C$. In general, $S t(C)=S t^{1}(C)$, and $S t^{K}(C)=S t\left(S t^{K-1}(C)\right)$.

Outline of Proof. Essentially one uses the cellular maps $\tilde{f}$ and $\widetilde{g}$ between $\tilde{X}$ and $\widetilde{Y}$ from Lemma 2.2 to define a 1-combing $\Lambda$ of $\tilde{Y}$ in terms of a given 1-combing $\Psi$ of $\widetilde{X}$. However, there is a bounded amount of adjustment necessary, primarily because $\widetilde{f} \circ \widetilde{g}(v) \neq v$. In addition to the constant $K$ mentioned in Lemma 2.2, the fact that $\widetilde{Y}$ is the universal cover of a finite PLCW-complex $Y$ can be used to find two other constants. There are fixed constants $M$ and $D$ such that if $v$ is any vertex of $\widetilde{Y}$ and $\xi$ is any loop of $\widetilde{Y}$ of length at most $K^{2}+2 K+1$ in $S t^{K+1}(v)$, then $\xi$ is homotopically trivial in $S t^{M}(v)$ and the corresponding homotopy $H_{\xi}$ can be 
assumed to be combinatorial with domain subdivided into at most $D$ cells. (We'll use this fact in the proof of Theorem B.)

Suppose $\Psi$ is a tame 1 -combing of $\tilde{X}$. Pick basepoints $\epsilon_{X} \in X^{0}$ and $\epsilon_{Y} \in Y^{0}$. Without loss of generality, as noted in Lemma 2.2, we may assume that $\tilde{f}\left(\epsilon_{X}\right)=\epsilon_{Y}$ and $\widetilde{g}\left(\epsilon_{Y}\right)=\epsilon_{X}$. For a vertex $v \in \widetilde{Y}^{0}, \widetilde{g}(v) \in \widetilde{X}^{0}$, so there is a 0-combing path $\left.\Psi\right|_{\widetilde{g}(v) \times[0,1]}$. The composition $\left.\tilde{f} \circ \Psi\right|_{\widetilde{g}(v) \times[0,1]}$ then gives a path from [0,1] to $\tilde{Y}^{1}$ connecting $\epsilon_{Y}$ to $\widetilde{f}(\widetilde{g}(v))$. Since $d_{Y}(\widetilde{f}(\widetilde{g}(v)), v) \leq K$, we can pick a path $\alpha_{v}$ in $\widetilde{Y}^{1}$ from $\widetilde{f}(\widetilde{g}(v))$ to $v$ of length at most $K$. Define $\left.\Lambda\right|_{v \times[0,1]}$ to be the path from $[0,1]$ to $\widetilde{Y}^{1}$ connecting $\epsilon_{Y}$ to $v$ by concatenating the paths $\left.\widetilde{f} \circ \Psi\right|_{\widetilde{g}(v) \times[0,1]}$ and $\alpha_{v}$; so $\Lambda(v, s)=\tilde{f}(\Psi(v, 2 s))$ when $0 \leq s \leq 1 / 2$ and $\Lambda(v, s)=\alpha_{v}\left(l\left(\alpha_{v}\right)(2 s-1)\right)$ when $1 / 2 \leq s \leq 1$.

The construction of the 1-combing is only slightly more complicated. Suppose $e$ is an edge of $\tilde{Y}$, and let $a$ and $b$ be the endpoints of $e$. Let $\gamma=\left\langle d_{1}, d_{2}, \ldots, d_{n}\right\rangle$ be the edge path $\widetilde{g}(e)$ in $\widetilde{X}$ from $\widetilde{g}(a)$ to $\widetilde{g}(b)$. Then $\widetilde{f}(\gamma)$ is a path in $\widetilde{X}$ from $\widetilde{f}(\widetilde{g}(a))=\alpha_{a}(0)$ to $\widetilde{f}(\widetilde{g}(b))=\alpha_{b}(0)$. Since $\Psi$ gives a combing of all of the edges of the path $\gamma,\left.\widetilde{f} \circ \Psi\right|_{\gamma \times[0,1]}$ gives a homotopy from $\tilde{f}(\gamma)$ to the image $\tilde{f}\left(\epsilon_{X}\right)=\epsilon_{Y}$ of the basepoint in $\widetilde{X}$. Every point of $\widetilde{f}(\gamma)$ is contained in $\widetilde{f}(\widetilde{g}(e))$, so each of these points is within a distance $K$ of a point of $e$. Then every point of the loop $\xi=\left\langle\alpha_{a}^{-1}, \widetilde{f}(\gamma), \alpha_{b}, e^{-1}\right\rangle$ must be in $S t^{K+1}(a)$, and the length of this loop is at most $K^{2}+2 K+1$. Let $H_{e}$ be the homotopy $H_{\xi}$ killing this loop in $S t^{M}(a)$.

Composing $H_{e}$ with a homeomorphism of $[0,1] \times[0,1]$ gives a homotopy $H_{e}^{\prime}$ with $H_{e}^{\prime}(s, 0)=\widetilde{f}(\gamma(s)), H_{e}^{\prime}(s, 1)=e(s), H_{e}^{\prime}(0, t)=\alpha_{a}(t)$, and $H_{e}^{\prime}(1, t)=\alpha_{b}(t)$. The homotopy $\Lambda: e \times[0,1] \rightarrow \widetilde{Y}$ is defined to be the homotopy $\left.\tilde{f} \circ \Psi\right|_{\gamma \times[0,1]}$ followed by $H_{e}^{\prime}$. This defines the 1 -combing $\Lambda$ of $\widetilde{Y}$. Since $\Psi$ is a tame combing of $\widetilde{X}$, Theorem 1 of [13] says that $\Lambda$ is a tame combing of $\tilde{Y}$.

Definition. Suppose $\rho: \mathbf{Q} \rightarrow \mathbf{Q}$ and $\rho^{\prime}: \mathbf{Q} \rightarrow \mathbf{Q}$ are functions. Define $\rho \preceq \rho^{\prime}$ if there is a positive constant $A$ such that $\rho(q) \leq A \rho^{\prime}(A q+A)+A$ for all $q \in \mathbf{Q}$. Then $\rho$ and $\rho^{\prime}$ are equivalent if both $\rho \preceq \rho^{\prime}$ and $\rho^{\prime} \preceq \rho$.

Theorem A. Suppose that $X$ and $Y$ are finite, connected 2-dimensional PLCWcomplexes whose fundamental groups are quasi-isometric. Let $\widetilde{X}$ and $\widetilde{Y}$ be their corresponding universal covers. Then $\tilde{X}$ has a tame 1-combing that admits a radial tameness function $\rho_{X}$ if and only if $\tilde{Y}$ has a tame 1-combing that admits an equivalent radial tameness function $\rho_{Y}$.

Proof. Suppose that $\widetilde{X}$ has a tame 1-combing $\Psi: \widetilde{X}^{1} \times[0,1] \rightarrow \widetilde{X}$ which admits a radial tameness function $\rho_{X}$. In the proof of Proposition 3.1, a tame 1-combing $\Lambda: \tilde{Y}^{1} \times[0,1] \rightarrow \widetilde{Y}$ for $\tilde{Y}$ is built using $\Psi$.

In order to find a radial tameness function $\rho_{Y}$ for $\Lambda$, we need two more constants. First, let $L$ be a positive constant such that for any vertex $v \in \widetilde{Y}^{0}$, the distance from $v$ to any point in $S t^{M}(v) \cap \widetilde{Y}^{1}$ is at most $L$, where $M$ is the constant defined in the proof of Proposition 3.1.

In our proof we move between levels of cells and the path metric on the Cayley graph. To facilitate this, if $x \in \widetilde{X}$, let $v_{x}$ denote any vertex on the boundary of the cell containing $x$; similarly, define $v_{y}$ to be a vertex in the boundary of the cell containing $y \in \tilde{Y}$. Define the constant $J$ to be 2 plus the maximum length 
of the boundary of any 2-cell in $X$ or $Y$. Then $J$ is greater than the difference in levels between any two cells for which one cell is in the boundary of the other, in both $\tilde{X}$ and $\tilde{Y}$. This shows that the differences $\left|\operatorname{lev}_{X}(x)-d_{X}\left(\epsilon_{X}, v_{x}\right)\right|<J$ and $\left|l e v_{Y}(x)-d_{Y}\left(\epsilon_{Y}, v_{y}\right)\right|<J$; hence moving between the level of an arbitrary point in $\widetilde{X}$ or $\tilde{Y}$, and the distance between a representative vertex and basepoint in the path metric on the 1-skeleton, introduces an easily constrained distortion. The third sentence of Lemma 2.2 implies that since the two points $x$ and $v_{x}$ in $\tilde{X}$ are contained in the same cell (including the boundary), their images $\widetilde{f}(x), \widetilde{f}\left(v_{x}\right) \in \widetilde{Y}^{1}$ are within $K$ cells of each other; hence $\left|l e v_{Y}(\widetilde{f}(x))-l e v_{Y}\left(\widetilde{f}\left(v_{x}\right)\right)\right|<K J$.

Let $C$ be a constant greater than $2 K+1,2 L+1$, and $2 K J$. Let $p=\Lambda(y, s)$ for some $y \in \widetilde{Y}^{1}$ with $y$ contained in an edge $e$ (possibly an endpoint of the edge). Suppose that $t>s$, and let $q=\Lambda(y, t)$, so $q$ is a point further along the same combing path as $p$. Assume that $l e v_{Y}(p)>C \rho_{X}\left(C n+7 C^{2}\right)+4 C^{2}$; it is our goal to show that the level of $q$ is greater than $n$, hence $\rho_{Y}=C \rho_{X}\left(C n+7 C^{2}\right)+4 C^{2}$ is a radial tameness function for $\Lambda$.

Case I. $p$ is in the image of $H_{e}^{\prime}$.

Then $q$ must also be in the image of $H_{e}^{\prime}$, which is contained in $S t^{M}(a)$ for an endpoint $a$ of $e$. Then from the definition of the constant $L, d_{Y}\left(a, v_{p}\right) \leq L$ and $d_{Y}\left(a, v_{q}\right) \leq L$, so $d_{Y}\left(v_{p}, v_{q}\right) \leq d_{Y}\left(a, v_{p}\right)+d_{Y}\left(a, v_{q}\right) \leq 2 L$. Then

$$
\begin{aligned}
\operatorname{lev} v_{Y}(q) & >d_{Y}\left(\epsilon_{Y}, v_{q}\right)-J \\
& \geq d_{Y}\left(\epsilon_{Y}, v_{p}\right)-d_{Y}\left(v_{p}, v_{q}\right)-J \geq d_{Y}\left(\epsilon_{Y}, v_{p}\right)-2 L-J \\
& >l e v_{Y}(p)-2 L-2 J>C \rho_{X}\left(C n+7 C^{2}\right)+4 C^{2}-2 C \\
& >n .
\end{aligned}
$$

Case II. $p$ and $q$ are both in the image of $\left.\tilde{f} \circ \Psi\right|_{\gamma \times[0,1]}$.

Then $p=\tilde{f}\left(\Psi\left(x, s^{\prime}\right)\right)$ and $q=\tilde{f}\left(\Psi\left(x, t^{\prime}\right)\right)$ for some $x \in \gamma$, with $s^{\prime}<t^{\prime}$.

Let $p^{\prime}=\Psi\left(x, s^{\prime}\right)$ and $q^{\prime}=\Psi\left(x, t^{\prime}\right)$.

Note that

$$
\begin{aligned}
l e v_{X}\left(p^{\prime}\right) & >d_{X}\left(\epsilon_{X}, v_{p^{\prime}}\right)-J \\
& \geq \frac{1}{K} d_{Y}\left(\widetilde{f}\left(\epsilon_{X}\right), \widetilde{f}\left(v_{p^{\prime}}\right)\right)-1-J=\frac{1}{K} d_{Y}\left(\epsilon_{Y}, \widetilde{f}\left(v_{p^{\prime}}\right)\right)-1-J \\
& =\frac{1}{K}\left(l e v_{Y}\left(\widetilde{f}\left(v_{p^{\prime}}\right)\right)\right)-1-J \\
& >\frac{1}{K}\left(l e v_{Y}\left(\widetilde{f}\left(p^{\prime}\right)\right)-K J\right)-1-J=\frac{1}{K}\left(l e v_{Y}(p)-K J\right)-1-J \\
& >\frac{1}{K}\left(C \rho_{X}\left(C n+7 C^{2}\right)+4 C^{2}-K J\right)-1-J \\
& >\rho_{X}\left(C n+7 C^{2}\right) .
\end{aligned}
$$

Since $p^{\prime}$ and $q^{\prime}$ are on the same combing path $\left.\Psi\right|_{\{x\} \times[0,1]}$, and $q^{\prime}$ is further along than $p^{\prime}$, the definition of the radial tameness function says that $l e v\left(q^{\prime}\right)>C n+7 C^{2}$. 
It follows that

$$
\begin{aligned}
\operatorname{lev}_{Y}(q) & =l e v_{Y}\left(\widetilde{f}\left(q^{\prime}\right)\right)>\operatorname{lev}\left(\widetilde{f}\left(v_{q^{\prime}}\right)\right)-K J \\
& =d_{Y}\left(\epsilon_{Y}, \widetilde{f}\left(v_{q^{\prime}}\right)\right)-K J=d_{Y}\left(\widetilde{f}\left(\epsilon_{X}\right), \widetilde{f}\left(v_{q^{\prime}}\right)\right)-K J \\
& \geq \frac{1}{K} d_{X}\left(\widetilde{g}\left(\widetilde{f}\left(\epsilon_{X}\right)\right), \widetilde{g}\left(\widetilde{f}\left(v_{q^{\prime}}\right)\right)\right)-1-K J \\
& \geq \frac{1}{K}\left(d_{X}\left(\epsilon_{X}, v_{q^{\prime}}\right)-d_{X}\left(\widetilde{g}\left(\widetilde{f}\left(\epsilon_{X}\right)\right), \epsilon_{X}\right)-d_{X}\left(\widetilde{g}\left(\widetilde{f}\left(v_{q^{\prime}}\right)\right), v_{q^{\prime}}\right)\right)-1-K J \\
& \geq \frac{1}{K}\left(d_{X}\left(\epsilon_{X}, v_{q^{\prime}}\right)-0-K\right)-1-K J \\
& >\frac{1}{K}\left(l e v_{X}\left(q^{\prime}\right)-J-K\right)-1-K J \\
& >\frac{1}{K}\left(C n+7 C^{2}-J-K\right)-1-K J \\
& >n+2 C .
\end{aligned}
$$

(We use the " $+2 C$ " in the next case.)

Case III. $p$ is in the image of $\left.\widetilde{f} \circ \Psi\right|_{\gamma \times[0,1]}$ and $q$ is in the image of $H_{e}^{\prime}$.

In this case we again have $p=\widetilde{f}\left(\Psi\left(x, s^{\prime}\right)\right)$ for some $x \in \gamma$ and $s^{\prime} \in[0,1]$. Let $r=\tilde{f}(\Psi(x, 1))=\widetilde{f}(x)$. Then $r=\Lambda(y, u)$ with $s \leq u \leq t$. Since $q$ and $r$ are both in the image of $H_{e}^{\prime}, d_{Y}\left(v_{q}, r\right) \leq 2 L$, as in Case I. Also, $p$ and $r$ are both in the image of $\left.\tilde{f} \circ \Psi\right|_{\gamma \times[0,1]}$, so Case II says that $\operatorname{lev}_{Y}(r)>n+2 C$. Then

$$
\begin{aligned}
\operatorname{lev}_{Y}(q) & >d_{Y}\left(\epsilon_{Y}, v_{q}\right)-J \\
& \geq d_{Y}\left(\epsilon_{Y}, r\right)-d_{Y}\left(r, v_{q}\right)-J \geq d_{Y}\left(\epsilon_{Y}, r\right)-2 L-J \\
& >l e v_{Y}(r)-2 L-2 J>n+2 C-2 L-2 J \\
& >n .
\end{aligned}
$$

Hence, in all three of the possible cases, if $\operatorname{lev}_{Y}(p)>C \rho_{X}\left(C n+7 C^{2}\right)+4 C^{2}$, then $\operatorname{lev}_{Y}(q)>n$. Therefore, the function $\rho_{Y}: \mathbf{Q} \rightarrow \mathbf{Q}$ defined by $\rho_{Y}(n)=$ $C \rho\left(C n+7 C^{2}\right)+4 C^{2}$ is a radial tameness function for the tame combing $\Lambda$ of $\widetilde{Y}$.

It is clear from the definition of $\rho_{Y}$ that $\rho_{Y} \preceq \rho_{X}$. Notice that $\rho_{X}(n)=$ $\frac{1}{C} \rho_{Y}\left(\frac{1}{C} n-7 C\right)-4 C$, so that $\rho_{X} \preceq \rho_{Y}$ also. Therefore the tame combing $\Lambda$ admits a radial tameness function equivalent to $\rho_{X}$.

Theorem B. Suppose that $X$ and $Y$ are finite, connected 2-dimensional $P L C W$ complexes whose fundamental groups are quasi-isometric. Let $\widetilde{X}$ and $\widetilde{Y}$ be their corresponding universal covers. Then $\widetilde{X}$ has a tame 1-combing that admits a ball tameness function $\beta_{X}$ if and only if $\widetilde{Y}$ has a tame 1-combing that admits an equivalent ball tameness function $\beta_{Y}$.

Proof. Let $B_{\tilde{X}}(n)$ and $B_{\widetilde{\widetilde{Y}}}(n)$ denote the balls of radius $n$ in $\widetilde{X}$ and $\widetilde{Y}$ respectively, and let $e$ be an edge of $\widetilde{Y}$. We need to bound the number of cells in the cellular decomposition of $e \times[0,1]$ that map to $B_{\widetilde{Y}}(r)$, using the ball tameness function $\beta_{X}$. In constructing $\Lambda: e \times[0,1]$, in the proof of Proposition 3.1, we used the edge path $\gamma=\left\langle d_{1}, d_{2}, \ldots, d_{n}\right\rangle$ which connected the $\widetilde{g}$-images of the end points of $e$. We then amalgamated the maps $\Psi: d_{i} \times[0,1]$, and used the map $\tilde{f}$ to send this amalgamation back to $\widetilde{Y}$. Now we just need to make a careful accounting of this process. 
Since the endpoints of $e$ are a distance one apart, the number $n$ of edges in the path $\gamma$ is $\leq K$, where $K$ is the constant in Lemma 2.2. Suppose $\sigma$ is a cell in any $d_{i} \times[0,1]$ that maps onto a cell $\tau$ in $e \times[0,1]$ which in turn maps into $B_{\widetilde{Y}}(r)$. Let $v_{\sigma}$ be a vertex on the boundary of $\sigma$. Then

$$
\begin{aligned}
l e v_{X}(\sigma) & <d_{X}\left(\epsilon_{X}, v_{\sigma}\right)+J \\
& \leq d_{X}\left(\epsilon_{X}, \widetilde{g}\left(\tilde{f}\left(v_{\sigma}\right)\right)\right)+d_{X}\left(\widetilde{g}\left(\widetilde{f}\left(v_{\sigma}\right)\right), v_{\sigma}\right)+J \\
& \leq d_{X}\left(\epsilon_{X}, \widetilde{g}\left(\widetilde{f}\left(v_{\sigma}\right)\right)\right)+K+J=d_{X}\left(\widetilde{g}\left(\widetilde{f}\left(\epsilon_{X}\right)\right), \widetilde{g}\left(\widetilde{f}\left(v_{\sigma}\right)\right)\right)+K+J \\
& \leq K d_{Y}\left(\widetilde{f}\left(\epsilon_{X}\right), \tilde{f}\left(v_{\sigma}\right)\right)+K+K+J=K d_{Y}\left(\epsilon_{Y}, \widetilde{f}\left(v_{\sigma}\right)\right)+2 K+J \\
& =K l e v_{Y}\left(\widetilde{f}\left(v_{\sigma}\right)\right)+2 K+J \\
& <K\left(l e v_{Y}(\tau)+K J\right)+2 K+J=\operatorname{Klev}_{Y}(\tau)+K^{2} J+2 K+J \\
& \leq K r+\left(K^{2} J+2 K+J\right) .
\end{aligned}
$$

Set $K^{\prime}=K^{2} J+2 K+J$ so that $\operatorname{lev}_{X}(\sigma)<K^{\prime} r+K^{\prime}$. Thus $\sigma$ has to map to $B_{\tilde{X}}\left(K^{\prime} r+K^{\prime}\right)$. By the definition of $\beta_{X}$, each $d_{i} \times[0,1]$ has a maximum of $\beta_{X}\left(K^{\prime} r+K^{\prime}\right)$ cells mapping to $B_{\tilde{X}}\left(K^{\prime} r+K^{\prime}\right)$. Because there are at most $K d_{i}$ 's, because each cell in each $d_{i} \times[0,1]$ maps to at most $K$ cells in $\tilde{Y}$ under $\tilde{f}$, and because the domain of each homotopy $H_{e}^{\prime}$ has at most $D$ cells (see the proof of Proposition 3.1), the total number of cells in $e \times[0,1]$ that could possibly map to $B_{\widetilde{Y}}(r)$ is bounded by $\beta_{Y}(r)=K^{2} \cdot \beta_{X}\left(K^{\prime} r+K^{\prime}\right)+D$.

\section{Almost convexity and linear Radial tameness}

In the introduction we noted that Cannon's almost convexity property is not geometric in that a group can be almost convex with respect to one set of generators and not with another. Here we show that an almost convex Cayley graph embeds as the 1-skeleton of a Cayley complex admitting the minimal possible radial tameness function $\rho(n)=n$. It follows that almost convex groups are contained in the quasiisometry class of groups admitting linear radial tameness functions.

While Cannon's definition of almost convexity was introduced in the context of Cayley graphs, it also applies to arbitrary graphs, in particular, to the 1-skeletons of simply connected PLCW-complexes.

Definition. For a 1-combing $\Psi$ of a 2-complex $\widetilde{X}$ and a point $x \in \tilde{X}$, the corresponding combing path $\left.\Psi\right|_{\{x\} \times[0,1]}$ is monotone increasing if whenever $s<t$, then either $\operatorname{lev}(\Psi(x, s))<\operatorname{lev}(\Psi(x, t))$, or $\operatorname{lev}(\Psi(x, s))=\operatorname{lev}(\Psi(x, t))$ and $\Psi(x,[s, t])$ is contained in the interior of a single cell of $\widetilde{X}$. The combing $\Psi$ is monotone increasing if all of its paths are monotone increasing.

Lemma 4.1. A 1-combing $\Psi$ is monotone increasing if and only if $\Psi$ is tame and admits a radial tameness function $\rho: \mathbf{Q} \rightarrow \mathbf{Q}$ given by $\rho(n)=n$.

Proof. Assume $\Psi$ is a monotone increasing 1-combing of $\tilde{X}$, and let $x \in \widetilde{X}^{1}$, $s \in[0,1], q \in \mathbf{Q}$, and $\operatorname{lev}(\Psi(x, s))>q$. Then for any point $\Psi(x, t)$ with $t>s$, $\operatorname{lev}(\Psi(x, t)) \geq \operatorname{lev}(\Psi(x, s))>q$ also, so $\Psi$ admits the radial tameness function $\rho(q)=q$.

On the other hand, if $\Psi$ admits a radial tameness function $\rho(q)=q$, if $s<t$, and if $\operatorname{lev}(\Psi(x, s)) \neq \operatorname{lev}(\Psi(x, t))$, then $\operatorname{lev}(\Psi(x, s))<\operatorname{lev}(\Psi(x, t))$. If $\operatorname{lev}(\Psi(x, s))=$ lev $(\Psi(x, t))$, but $\Psi(x, s)$ and $\Psi(x, t)$ aren't contained in a single open cell, then 
the path $\Psi(x,[s, t])$ must cross an open cell $\sigma$ whose dimension is different from the dimension of the open cell containing $\Psi(x, s)$. But cells of different dimensions can't have the same level, so by the fact that $\rho(q)=q, \operatorname{lev}(\sigma)>\operatorname{lev}(\Psi(x, s))$. Again by the fact that $\rho(q)=q$ this implies that it is impossible for $\operatorname{lev}(\Psi(x, s))=$ $\operatorname{lev}(\Psi(x, t))$.

Theorem C. A finitely presented group $G$ is almost convex with respect to a finite set of generators if and only if there is a finite presentation, with the same set of generators, such that the associated Cayley complex admits a monotone increasing tame 1-combing.

Theorems A and C, along with Lemma 4.1, immediately imply the following corollary. (See Corollary 4.3 also.)

Corollary 4.2. The class of almost convex groups is contained in the presentation independent class of groups admitting tame 1-combings with linear radial tameness functions.

Proof of Theorem C. $(\Rightarrow)$ For this direction we revisit the construction of the tame 1-combing for an almost convex group given in [6]; the general technique outlined here is also used in the proof of Theorem D.

Suppose that $G$ is almost convex with respect to a finite generating set $S$. Let $A$ be the corresponding almost convexity constant. Let $R$ be any finite set of defining relators for $G$ that contains every word of length up to $2 A+2$ that represents the trivial element of $G$. Finally, let $\widetilde{X}$ be the Cayley 2-complex associated with the presentation given by $S$ and $R$.

Define a 0 -combing $\Psi: \widetilde{X}^{0} \times[0,1] \rightarrow \widetilde{X}$ by taking the combing path $\left.\Psi\right|_{\{v\} \times[0,1]}$ for any vertex $v$ to be the path (without backtracking) of a geodesic in $\widetilde{X}^{1}$ from $\epsilon$ to $v$. Moreover, choose the paths in this 0 -combing so that for any vertex $x$ on a combing path $\left.\Psi\right|_{\{v\} \times[0,1]}$, the 0-combing path $\left.\Psi\right|_{\{x\} \times[0,1]}$ for $x$ is simply the portion of $\left.\Psi\right|_{\{v\} \times[0,1]}$ that goes from the basepoint to $x$, reparametrized; that is, choose the 0 -combing to be prefix closed. (For example, the 0-combing given by taking the paths corresponding to the shortlex minimal representatives of each of the group elements has this property.) With this choice, each of the 0-combing paths are monotone increasing.

We define the combing $\left.\Psi\right|_{e \times[0,1]}$ for an edge $e$ by induction on the level of the edge. Note that with our assumption on presentations in $\S 2.1$, no generator has order 1 , so there is no edge with level $\frac{1}{4}$. Suppose that $e$ is an edge with level $\frac{3}{4}$. Then for any point $x$ in the edge $e$, define the combing path $\left.\Psi\right|_{\{x\} \times[0,1]}$ to be a path that runs directly from the endpoint $\epsilon$ of $e$ to the point $x$. Next suppose that $e$ is an edge with level $\frac{5}{4}$. Then the endpoints $a$ and $b$ of $e$ are connected to the basepoint $\epsilon$ by single edges $c$ and $d$, respectively. These three edges $\langle c, e, d\rangle$ must therefore form a loop in $\widetilde{X}^{1}$. The definition of $R$ implies that there is a 2-cell in $\widetilde{X}$ whose boundary is given by this loop. In this case define the homotopy $\left.\Psi\right|_{e \times[0,1]}$ to fill in this 2-cell.

Next suppose that the combing $\Psi$ has been defined for all points in $\widetilde{X}^{1}$ with level less than $n+\frac{1}{4}$ for $n \in \mathbf{N}$, and that all of the combing paths for these points are monotone increasing.

Let $e$ be an edge at level $n+\frac{1}{4}$ with $n \in \mathbf{N}$. Then the endpoints $a$ and $b$ of $e$ are both in the sphere $S(n)$, and $d(a, b)=1$ (since we assume no generator has order $1)$. The definition of almost convexity then says that there is a path $\alpha$ of length at 
most $A$ inside of $B(n)$ from $a$ to $b$. Note that since all of the points on this path are inside of $B(n)$, the level of all of the points on this path is at most $n$, also. By induction, the combing $\Psi$ has been defined on all of the points of the path $\alpha$. The path $\alpha$ together with the edge $e$ form a loop in $\widetilde{X}$ of length at most $A+1$. The definition of the set of relators then says that there must be a 2-cell in $\widetilde{X}$ whose boundary is given by $\alpha$ and $e$. Define the combing $\left.\Psi\right|_{e \times[0,1]}$ to be the combing of $\alpha$ followed by a homotopy filling in the 2-cell. Since the combing of $\alpha$ is monotone increasing, and all of the points of $\alpha$ have level less than $n+\frac{1}{4}$, the combing for $e$ is also monotone increasing.

Finally, suppose that $e$ is an edge at level $n+\frac{3}{4}$ with $n \in \mathbf{N}$. The edge $e$ has an endpoint $a$ with $\operatorname{lev}(a)=n$ and an endpoint $b$ with $\operatorname{lev}(b)=n+1$. The combing path $\left.\Psi\right|_{\{b\} \times[0,1]}$ follows a geodesic in $X^{1}$ from $\epsilon$ to $b$, so it must go through a point $c$ with $\operatorname{lev}(c)=n$. The distance from $a$ to $c$ is at most 2. If $a=c$, then for any point $x$ of $e$, define the combing path to $x$ to be the portion of the combing path $\left.\Psi\right|_{\{b\} \times[0,1]}$ from the basepoint to $x$, reparametrized. If $1 \leq d(a, c) \leq 2$, then almost convexity implies that there is a path $\alpha$ from $a$ to $c$ inside of $B(n)$ of length at most $A$. As in the paragraph above, define $\left.\Psi\right|_{e \times[0,1]}$ to be the combing of $\alpha$ followed by a homotopy filling in the 2-cell bounded by $\alpha$, the edge from $c$ to $b$, and $e$; this is again monotone increasing.

$(\Leftarrow)$ Suppose that $G$ has a finite presentation for which the corresponding Cayley complex $\widetilde{X}$ has a monotone increasing tame 1-combing $\Psi$. Since $\widetilde{X}$ is the universal cover of a finite complex, there is a constant $C$ such that the number of edges in $S t^{2}(e)$ for any edge $e$ is bounded by $C$; we will show that $G$ is almost convex with constant $A=2 C$.

Suppose that $g, h \in S(n)$ and $d(g, h) \leq 2$. The case where $d(g, h)=0$ is trivial.

Case I. $d(g, h)=1$.

In this case $g$ and $h$ are joined by an edge $e$ of level $n+\frac{1}{4}$. Since the map $\left.\Psi\right|_{e \times[0,1]}$ is combinatorial, there are finitely many 2 -cells in the domain whose closure intersects $e \times\{1\}$; all such cells map into the first cellular neighborhood of $e \in \widetilde{X}$. We call this collection $\Upsilon$. Because the level of $e$ is $n+\frac{1}{4}$, and $\Psi$ is monotone increasing, the level of any vertex in the image of $\Upsilon$ is at most $n$, and the level of any edge in $\Psi(\partial \Upsilon)-\{e\}$ must be less than $n+\frac{1}{4}$ and hence is at most $n-\frac{1}{4}$. So the path $\Psi(\partial \Upsilon)-\{e\}$ is a path joining $g$ and $h$ in $B(n)$. By eliminating loops in this path, $g$ can be joined to $h$ by a path of length at most $C$ which stays inside of $S t(e) \cap B(n)$.

Case II. $d(g, h)=2$.

Let $d$ and $e$ be a path of length 2 from $g$ to $h$ in $\widetilde{X}^{1}$, and let $f$ be their common endpoint. If $\operatorname{lev}(f)=n-1$, then $d$ and $e$ are a path of length 2 in $B(n)$ from $g$ to $h$. If $\operatorname{lev}(f)=n$, then applying Case I to the edges $d$ and $e$ shows that there is a path of length at most $2 C$ inside $B(n)$ from $g$ to $h$.

Now suppose that $\operatorname{lev}(f)=n+1$. Because the 1 -combing restricts to a 0 -combing on the endpoints of edges, the two maps $\left.\Psi\right|_{d}$ and $\left.\Psi\right|_{e}$ can be combined into one monotone increasing map $\Psi:\{d \cup e\} \times[0,1] \rightarrow X$. Again we will take a cellular subdivision of this map which makes it combinatorial, and let $\Upsilon$ be the collection of 2-cells in $\{d \cup e\} \times[0,1]$ whose closure intersects $d, e$ (or $f$ ). Then $\Upsilon$ maps under $\left.\Psi\right|_{d \cup e}$ to the first cellular neighborhood of $\{d \cup e\}$, which is the union of the first cellular neighborhoods of $d$ and $e$. As a first approximation, we look at the path 
$\Psi(\partial \Upsilon)-\{d, e\}$ joining $g$ to $h$. Because $\Psi$ is monotone increasing, the level of any edge in $\Psi(\partial \Upsilon)-\{d, e\}$ is $<n+\frac{3}{4}$ and hence is $\leq n+\frac{1}{4}$.

Let $\sigma$ be a 2 -cell in $\Upsilon$ that contains an edge $e^{\prime}$ with $\operatorname{lev}\left(\Psi\left(e^{\prime}\right)\right)=n+\frac{1}{4}$. Case I shows how the endpoints of $\Psi\left(e^{\prime}\right)$ can be connected by an edge path in $B(n)$. Replacing all edges of level $n+\frac{1}{4}$ in $\Psi(\partial \Upsilon)-\{d, e\}$ in this way yields a path from $g$ to $h$ contained in $B(n)$. By construction this path is contained in the union of the second cellular neighborhoods of $d$ and $e$, and hence, after removing loops, its length is bounded by $2 C$.

There are two weaker versions of almost convexity for groups in the literature. One was introduced by Poénaru in [15]. A group $G$ is $P$-almost convex if it satisfies two conditions. Poénaru's condition $1^{\circ}$ is a variation on a condition of Casson and it implies that the group admits a linear isodiametric function. Condition $2^{\circ}$ is an almost convexity condition: there are positive constants $C$ and $\epsilon$ (where $0<\epsilon<1$ ) such that for any integer $n$ and any pair of group elements $g, h \in G$ with $g, h \in S(n)$ and $d(g, h) \leq 3$, there is a path in $B(n)$ of length at most $C n^{1-\epsilon}+C$ joining $g$ and $h$.

Poénaru and Tanasi introduced another variation on Cannon's almost convexity condition using isoperimetric functions [16. Suppose that $k$ is a natural number and that $g$ and $h$ are arbitrary elements of $S(n)$ for some $n$ with $d(g, h) \leq k$. Let $p_{1}$ be a path in $\widetilde{X}^{1}$ from $g$ to $h$ of length at most $k$, and let $p_{2}$ be any path inside of $B(n)$ from $h$ to $g$. This gives a closed loop in $\widetilde{X}^{1}$, which must bound a disk in $\widetilde{X}$. Among all disks bounded by loops formed by paths with these properties, let $D$ be a disk for which the number of 2-cells in $D$, or the area of $D$, is minimal. If there are positive constants $C$ and $\epsilon$, depending only on $k$, such that $\operatorname{area}(D) \leq C n^{1-\epsilon}+C$ for all $n \in \mathbf{N}$ and all $g, h \in S(n)$ with $d(g, h) \leq k$, then the group is said to be $k$-weakly almost convex. The group is weakly almost convex if it is $k$-weakly almost convex for every natural number $k$.

Theorem D. If a group $G$ is either P-almost convex or weakly almost convex with respect to a finite presentation, then the corresponding Cayley 2-complex has a tame combing with a linear radial tameness function.

Using Theorem D we can extend Corollary 4.2 to

Corollary 4.3. The quasi-isometry invariant class of groups admitting tame 1combings with linear radial tameness functions contains all almost convex, P-almost convex, and weakly almost convex groups.

Proof of Theorem D. The proofs in both cases are essentially the same, starting with the construction of a 1-combing, similar to the one made in the case of almost convexity.

Suppose that $G$ is P-almost convex, so that $\left(1^{\circ}\right) G$ admits a linear isodiametric function, and $\left(2^{\circ}\right)$ there are positive constants $C$ and $\epsilon$ (where $\epsilon<1$ ), such that whenever $a, b \in S(n)$ and $d(a, b) \leq 3$, there is an edge path contained in $B(n)$ of length at most $C n^{1-\epsilon}+C$ joining $a$ and $b$.

The 0 -combing is constructed by taking any geodesic, prefix closed combing from a chosen basepoint. The 1-combing is constructed inductively much as in the case of Cannon's almost convexity. Let $e$ be an edge with vertices $a$ and $b$. If the level of $e$ is $n+\frac{1}{4}$, then $\operatorname{lev}(a)=\operatorname{lev}(b)=n$ and $a \neq b$, hence by condition $2^{\circ}$ there is an edge-path joining $a$ to $b$ contained in $B(n)$, of length $<C n^{1-\epsilon}+C$. Similarly, 
if $\operatorname{lev}(e)=n+\frac{3}{4}$, then without loss of generality we may assume $\operatorname{lev}(a)=n$ and $\operatorname{lev}(b)=n+1$, and that the penultimate vertex $c$ in the combing path for $b$ is at level $n$. Then there exists an edge path from $a$ to $c$ contained in $B(n)$, of length $<C n^{1-\epsilon}+C$. Call the edge path in $B(n)$ the $A C$ path associated with the edge $e$.

If $\operatorname{lev}(e)=n+\frac{1}{4}$, or $\operatorname{lev}(e)=n+\frac{3}{4}$ and $a \neq c$, then the union of $e$, the AC path for $e$, and possibly the edge $\{b, c\}$ when $\operatorname{lev}(e)=n+\frac{3}{4}$, forms a loop in $\widetilde{X}$. In the "classical" setting of Cannon's almost convexity, these loops had bounded length, hence we could assume they were filled by a single 2-cell. Passing to the more general setting, we still have reasonable control over how to fill these loops in order to construct a tame 1-combing for $\widetilde{X}$. By condition $1^{\circ}$ (which implies there is a linear bound on the diameter of filling disks in terms of the length of the perimeter), one can fill this loop such that every vertex in the filling is at most a distance $C^{\prime} n^{1-\epsilon}+C^{\prime}$ from $a$ or $b$, where $C^{\prime}$ is some constant greater than $C$. Call such a filling the $A C$ bead associated to the points of the edge $e$. Note that every edge in the $\mathrm{AC}$ path for $e$ is at a lower level than $e$. Thus, by induction, there are 1-combing paths out to all of the edges of the $\mathrm{AC}$ path for $e$. The 1-combing for $e$ is constructed via concatenating the 1-combings to the edges in the AC path for $e$, and then adding a homotopy from the $\mathrm{AC}$ path for $e$, to $e$, passing through the AC bead.

It may occur that $\operatorname{lev}(e)=n+\frac{3}{4}$ and $a=c$. In this case then the AC path for $e$ consists of a single point. The 1-combing path to any point $p$ on the edge $e$ is defined to be the 0 -combing path from the basepoint to $a$, followed by the portion of the edge $e$ from $a$ to $p$. The portion of the edge $e$ in this path is called the $A C$ bead for the point $p$; the $\mathrm{AC}$ bead for the vertex $b$ is the entire edge $e$.

Ultimately, each combing path $\left.\Psi\right|_{\{x\} \times[0,1]}$ will run through a necklace of AC beads as it moves from the basepoint to the edge $e$. Thus there is a finite sequence of special points $\left\{p_{1}, \ldots, p_{j}, x\right\}$ where the combing path begins at the basepoint, stays inside an $\mathrm{AC}$ bead until it reaches the point $p_{1}$ where it passes into a second $\mathrm{AC}$ bead, the AC bead associated with $p_{2}$. The combing path exits this second $\mathrm{AC}$ bead at the point $p_{2}$, then travels through the third $\mathrm{AC}$ bead until it reaches $p_{3}$, etc., until the final AC bead bridges the gap between $p_{j}$ and the point being 1-combed, $x$. Note that because $p_{i-1}$ is part of the AC path for $p_{i}$, the level of $p_{i-1}$ is strictly less than the level of $p_{i}$.

Every vertex in the $n$th $\mathrm{AC}$ bead is a distance at most $C^{\prime} m^{1-\epsilon}+C^{\prime}+J$ from $p_{n}$, where the level of $p_{n}$ is $m$. Setting $C^{\prime \prime}=C^{\prime}+2 J$ we see that every cell in the $n$th $\mathrm{AC}$ bead has level at least $m-C^{\prime \prime} m^{1-\epsilon}-C^{\prime \prime}$ and at most $m+C^{\prime \prime} m^{1-\epsilon}+C^{\prime \prime}$. Because $C^{\prime \prime} m^{1-\epsilon}+C^{\prime \prime}$ is sublinear, there is a constant $K$ such that

$$
C^{\prime \prime} m^{1-\epsilon}+C^{\prime \prime}<\frac{1}{3} m+K
$$

for all $m \geq 0$. Thus if $l$ is the level of any cell in the AC bead for $p_{n}$, we get

$$
\frac{2}{3} m-K<l<\frac{4}{3} m+K
$$

hence

$$
\frac{3}{4}(l-K)<m<\frac{3}{2}(l+K) .
$$

Set $\rho(q)=2 q+3 K$. Assume that a combing line passes through a cell whose level is $>2 q+3 K$, and let $p_{n}$ be the special point whose AC bead contains that cell. 
Then the level of $p_{n}$ is $>\frac{3}{4}(2 q+2 K)=\frac{3}{2}(q+K)$. But then any cell in this AC bead has level $>\frac{2}{3}\left[\frac{3}{2}(q+K)\right]-K=q$. Further, because the levels of the special points are strictly increasing, and because the level of the point $p_{n}$ is $>\frac{3}{2}(q+K)$, the level of any special point $p_{j}(j>n)$ must also be $>\frac{3}{2}(q+K)$. So the level of any cell in the $j$ th AC bead $(j>n)$ is also at least $q$.

Weakly Almost Convex. We use the same construction as in the almost convex and $\mathrm{P}$-almost convex cases to construct the tame combing: Comb out to the vertices using prefix closed geodesics; connect the two vertices on an edge by a path in $B(n)$; fill the loop created in a minimal manner. The weak almost convexity criterion states that this filling has area $\leq C n^{1-\epsilon}+C$. Note that the diameter of this filling is then at most $J C n^{1-\epsilon}+J C$, where $J$ is the length of the longest relator. Further, the length of the path in $B(n)$ is also bounded by $J C n^{1-\epsilon}+J C$. Thus the two key ingredients from the previous case still hold, and the argument follows mutatis mutandis.

Note. Mihalik and Tschantz showed that if $M$ is a closed irreducible 3-manifold with an infinite fundamental group that is tame combable, then the universal cover of $M$ is homeomorphic to $\mathbf{R}^{3}$ [13]. In [6] we showed that if $\pi_{1}(M)$ satisfies Cannon's almost convexity condition, then $\pi_{1}(M)$ admits a tame combing, hence by Mihalik and Tschantz, the covering conjecture holds for $M$. (This is the main result of [14.) It follows from Theorem $\mathrm{D}$ that this is true if $\pi_{1}(M)$ is $\mathrm{P}$-almost convex or weakly almost convexity. This gives a new proof of the main results of [15] and [16].

\section{Examples of groups Without linear RADial tame COMBings}

In $\S 6$ of [4], Gersten studied the iterated HNN extensions

$$
G_{n}=\left\langle a_{0}, a_{1}, \ldots, a_{n} \mid a_{i}^{a_{i+1}}=a_{i}^{2} ; 0 \leq i \leq n-1\right\rangle
$$

and showed that they have isoperimetric functions bounded below by iterated exponential functions. In this section we show that these groups admit finite complete rewriting systems, hence they are tame combable, but they cannot have tame combings with linear radial tameness functions.

Recall that a rewriting system consists of a finite set $\Sigma$ and a subset $R \subseteq \Sigma^{*} \times \Sigma^{*}$ of replacement rules, written $u \rightarrow v$ whenever $(u, v) \in R$, which give, for any $x, y \in \Sigma^{*}$, rewritings xuy $\rightarrow$ xvy. This is a rewriting system for a group $G$ if

$$
\langle\Sigma| u=v \text { if }(u, v) \in R\rangle
$$

is a presentation for $G$ as a monoid. The rewriting system is finite if the sets $\Sigma$ and $R$ are finite, and the system is complete if the following conditions hold:

(C1) There is no infinite chain $x \rightarrow x_{1} \rightarrow x_{2} \rightarrow \cdots$ of rewritings.

(C2) There is exactly one word representing each element of the monoid presented by the rewriting system that cannot be rewritten.

The rewriting system is geodesic if all of the rules either decrease or preserve word length. 
Note that Theorem B of [6] says that if a group has a geodesic finite complete rewriting system, then the group is almost convex with the same set of generators. The following corollary then follows directly from Theorem $\mathrm{C}$ above.

Corollary 5.1. If a group $G$ has a geodesic finite complete rewriting system, then the corresponding tame 1-combing is monotone increasing.

Proposition 5.2. The groups $G_{n}$ admit finite complete rewriting systems.

Theorem A in [6] states that any group admitting a finite complete rewriting system is tame 1-combable. Hence we immediately get the following.

Corollary 5.3. The groups $G_{n}$ are tame 1-combable.

We note that Gersten suggested (in a note to C.F. Miller, III) that the groups $G_{n}$ ought to admit finite complete rewriting systems, and proposed such a presentation of $G_{3}$. However, the general result for $G_{n}$, as well as the proof, is new.

Proof of Proposition 5.2. For the groups $G_{n}$, define the generating set

$$
\Sigma_{n}=\left\{a_{0}, A_{0}, a_{1}, A_{1}, \ldots, a_{n}, A_{n}\right\} .
$$

Let $R_{n}$ consist of the rewritings $a_{i} A_{i} \rightarrow 1$ and $A_{i} a_{i} \rightarrow 1$ for each $0 \leq i \leq n$, along with the rules

$$
\begin{aligned}
a_{k-1} a_{k} & \rightarrow A_{k-1} a_{k} a_{k-1}, \\
a_{k-1} A_{k} & \rightarrow A_{k} a_{k-1} a_{k-1}, \\
A_{k-1} A_{k-1} a_{k} & \rightarrow a_{k} A_{k-1} \\
A_{k-1} A_{k} & \rightarrow A_{k} A_{k-1} A_{k-1}
\end{aligned}
$$

when $1 \leq k \leq n$ and $k$ is odd, and the rules

$$
\begin{aligned}
a_{k+1} a_{k} & \rightarrow a_{k} a_{k} a_{k+1}, \\
a_{k+1} A_{k} & \rightarrow A_{k} A_{k} a_{k+1}, \\
A_{k+1} a_{k} a_{k} & \rightarrow a_{k} A_{k+1}, \\
A_{k+1} A_{k} & \rightarrow A_{k} A_{k+1} a_{k}
\end{aligned}
$$

when $1 \leq k \leq n-1$ and $k$ is again odd. This is a rewriting system for $G_{n}$ since it gives a monoid presentation for this group.

To check that condition (C1) holds, it suffices to show that there is a well-ordering compatible with multiplication, or reduction ordering, which is reduced by each of the rules in $R$. We define the ordering recursively, by iterating the wreath product ordering (see pp. 46-50 in [17] for details). First, for each $1 \leq k \leq n$ with $k$ odd, define the ordering $\ll_{k}$ to be the ordering on the set of words $\left\{a_{k}, A_{k}\right\}^{*}$ given by the shortlex ordering with $A_{k}>a_{k}$. For each $0 \leq j \leq n$ with $j$ even, define the ordering $\ll_{j}$ to be the ordering on the set of words $\left\{a_{j}, A_{j}\right\}^{*}$ given by the shortlex ordering with $a_{j}>A_{j}$.

Next define the ordering $<_{0}$ on the set $\Sigma_{0}^{*}$ of words over $\Sigma_{0}=\left\{a_{0}, A_{0}\right\}$ to be $<_{0}=\ll_{0}$. The ordering $<_{1}$ on the set $\Sigma_{1}^{*}$ of words over $\Sigma_{1}=\left\{a_{0}, A_{0}, a_{1}, A_{1}\right\}$ is given by taking the left-to-right wreath product ordering $<_{1}=<_{0} 2 \ll_{1}$. In this ordering, in order to compare two words $u$ and $v$, write $u=S_{0} b_{1} S_{1} b_{2} \ldots S_{r-1} b_{r} S_{r}$ and $v=T_{0} d_{1} T_{1} d_{2} \ldots T_{p-1} d_{p} T_{p}$ with $S_{i}, T_{i} \in\left\{a_{0}, A_{0}\right\}^{*}$ and $b_{i}, d_{i} \in\left\{a_{1}, A_{1}\right\}$. Then $u<_{1} v$ if one of the following holds:

(i) $b_{1} b_{2} \ldots b_{r} \ll_{1} d_{1} d_{2} \ldots d_{p}$ 
(ii) $b_{1} b_{2} \ldots b_{r}=d_{1} d_{2} \ldots d_{p}$ and $\left(S_{0}, S_{1}, \ldots, S_{r}\right)$ comes before $\left(T_{0}, T_{1}, \ldots, T_{r}\right)$ in the left-to-right (i.e., usual) lexicographic ordering of $\left(\Sigma_{0}^{*}\right)^{r+1}$ defined by $<_{0}$.

Since $<_{0}$ and $\ll_{1}$ are both reduction orderings, Proposition 1.7, p. 47 of [17] shows that $<_{1}$ is also a reduction ordering.

The ordering $<_{2}$ on the set $\Sigma_{2}^{*}$ is defined by taking the right-to-left wreath product ordering $<_{2}=<_{1} \imath^{\prime} \ll_{2}$. For two words $u=S_{0} b_{1} S_{1} b_{2} \ldots S_{r-1} b_{r} S_{r}$ and $v=$ $T_{0} d_{1} T_{1} d_{2} \ldots T_{p-1} d_{p} T_{p}$ with $S_{i}, T_{i} \in \Sigma_{1}^{*}$ and $b_{i}, d_{i} \in\left\{a_{2}, A_{2}\right\}, u<_{2} v$ if one of the following holds:

(i) $b_{1} b_{2} \ldots b_{r} \ll_{2} d_{1} d_{2} \ldots d_{p}$

(ii) $b_{1} b_{2} \ldots b_{r}=d_{1} d_{2} \ldots d_{p}$ and $\left(S_{0}, S_{1}, \ldots, S_{r}\right)$ comes before $\left(T_{0}, T_{1}, \ldots, T_{r}\right)$ in the right-to-left lexicographic ordering of $\left(\Sigma_{1}^{*}\right)^{r+1}$ defined by $<_{1}$.

A slight variation on Proposition 1.7 in [17] again shows that $<_{2}$ is a reduction ordering, since $<_{1}$ and $\ll_{2}$ are.

Repeat this procedure to define an ordering $<_{k}$ on $\Sigma_{k}^{*}$ for any $k$. If $k$ is odd, then let $<_{k}=<_{k-1} \imath \ll_{k}$, and if $k$ is even, then let $<_{k}=<_{k-1} \imath^{\prime} \ll_{k}$. In each case, the ordering $<_{k}$ is a reduction ordering.

It is straightforward to check that this rewriting system is compatible with this iterated wreath product ordering; that is, for each of the rewritings $u \rightarrow v \in R_{n}$, $v<_{n} u$. Thus the rewriting system satisfies condition (C1). The Knuth-Bendix procedure $[8$ is a procedure which is used to check that the condition (C2) holds for a rewriting system in which $(\mathrm{C} 1)$ is already known to hold. This is again a straightforward computation for the rewriting system given by $\Sigma_{n}$ and $R_{n}$; therefore this rewriting system is finite and complete.

Proposition 5.4. The groups $G_{n}$ do not admit tame 1-combings with linear radial tameness functions.

Proof. Gersten uses a Koch snowflake picture to demonstrate that $G_{n}$ has an isoperimetric function bounded below by an iterated exponential function ( $\S 6$ of [4] again). In particular, he shows that the isoperimetric function for $G_{n}$ grows at least as fast as $E_{n}$ where

$$
E_{n}(l):=\underbrace{2^{2 \cdot \cdot^{2^{l}}}}_{n \text { times }} .
$$

The Koch snowflake picture is a reduced van Kampen diagram for the nullhomotopic word

$$
\omega_{n, l}=\left[a_{0}^{a_{1}^{\cdot{ }^{a_{n}^{l}}}}, a_{0}\right]
$$

of length $2^{n+1}(l+1)$ that is filled by more than $E_{n}(l) 2$-cells. Because $G$ is aspherical [4], any filling of the null-homotopic word $\omega_{n, l}$ must involve all the cells in Gersten's Koch snowflake diagram, and this van Kampen diagram embeds in the Cayley complex $\widetilde{X}$. Suppose the image of the van Kampen diagram is contained in the ball $B(r)$. Since the number of vertices in $B(r) \subset \widetilde{X}$ is at most $(2(n+1))^{r}$, and the diagram contains at least $E_{n}(l)$ vertices, $r$ must be at least $E_{n-1}(l) / \log _{2}(2(n+1))=$ $O\left(E_{n-1}(l)\right)$. Then the maximum distance from the identity to a vertex in this filling of $\omega_{n, l}$ must be at least $O\left(E_{n-1}(l)\right)$. Thus all fillings of $\omega_{n, l}$ contain vertices a distance at least $O\left(E_{n-1}(l)\right)$ from $\epsilon$ in the Cayley graph. 


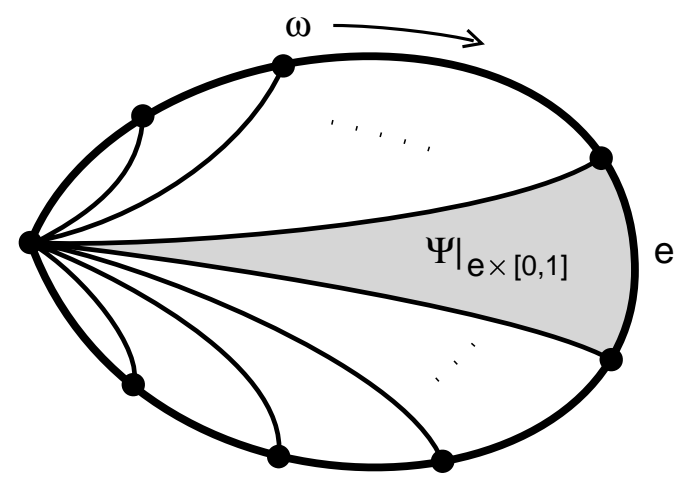

Figure 1. The sea shell filling of a null homotopic loop.

Tame 1-combings can be used to fill null homotopic words. If $\omega={ }_{G} 1$, then for each edge $e \subset \omega,\left.\Psi\right|_{e \times[0,1]}$ gives a homotopy from $\epsilon$ to $e$. The union

$$
\bigcup\left\{\left.\Psi\right|_{e \times[0,1]} \mid e \in \omega\right\}
$$

where one amalgamates $\left.\Psi\right|_{e \times[0,1]}$ and $\left.\Psi\right|_{e^{\prime} \times[0,1]}$ along $\left.\Psi\right|_{a \times[0,1]}$ when $e \cap e^{\prime}=a$, is a disk filling in $\omega$. We call this the sea shell filling of $\omega$ (see Figure 1).

Suppose the group $G_{n}$ admits a tame combing with linear radial tameness function $\rho_{n}(q)=K_{n} q+K_{n}$ for some constant $K_{n}$. If the length of $\omega$ is $l$, then the distance (in the Cayley graph) from $\epsilon$ to any vertex in the corresponding sea shell filling is bounded by $\rho(l)$. That is, the image of this disk in $\widetilde{X}$ is contained in $B(\rho(l))$. Then one could fill the Koch snowflake loop $\omega_{n, l}$ in $B\left(K_{n} l+K_{n}\right)$ for all $l$. But as we noted above, these Koch snowflake loops can only be filled in $B(r)$, for some $r=O\left(E_{n-1}(l)\right)$ (or larger). Hence these groups cannot admit linear radial tameness functions, and moreover, their radial tameness functions are bounded from below by iterated exponentials.

\section{ISOPERIMETRIC AND ISODIAMETRIC FUNCTIONS AND SOLVABILITY OF THE WORD PROBLEM}

Given a group $G$ and a finite set of generators for $G$, Dehn's word problem asks if there is an algorithm which can determine, given any word in the generators, whether or not the word represents the trivial element of the group. This problem is not solvable in general; that is, there are finitely presented groups for which there is no such algorithm. (See [9] for details.) In this section we show that if the measurements for a tame combing for $G$ are recursive, or computable, then the word problem is solvable. In particular, we establish that the isodiametric and isoperimetric functions are recursive, or computable.

In the previous section we studied the diameters of images of van Kampen diagrams in the Cayley complex in order to establish that there are tame combable groups whose radial tameness functions are at least multiply exponential. Given this, one might imagine that there is a tight connection between isodiametric functions and radial tameness functions. However, the connection is not clear. For example, given any null homotopic word $\omega$ one can use a tame 1-combing to form a van Kampen diagram for $\omega$ using the sea shell method. As noted in $\S 5$, this van 
Kampen diagram is mapped to $B(\rho(l(\omega))) \subset \widetilde{X}$. In the examples of $\S 5$ we had the added advantage of knowing that these van Kampen diagrams embed in $\widetilde{X}$, but this is not true in general. Cells in $B(\rho(l(\omega))) \subset \widetilde{X}$ could be hit multiple times; hence there is no immediate way to bound either the combinatorial diameter or area of such disks.

Conversely, let $g: \mathbf{N} \rightarrow \mathbf{N}$ be an isodiametric function for $G$. One can make a 1-combing as follows: Let the 0-combing consist of geodesics from $\epsilon$ to each $v \in \widetilde{X}^{0}$. Each edge $e$ determines a 0 -combing loop consisting of $e$ and the 0 -combing paths to the vertices of $e$. Have the 1-combing for $e$ pass through a filling of this 0combing loop that satisfies the isodiametric condition given by $g$. It is true that if lev $(e)=n \pm \frac{1}{4}$, then no combing line for a point in $e$ will leave $B(g(2 n)+J)$, so one might be tempted to believe $\rho(n):=g(2 n)+J$ would be a radial tameness function for $G$. However, if $\operatorname{lev}(e) \gg n$, there is no reason to assume that once a combing line for a point in $e$ leaves $B(g(2 n)+J)$ it won't return to $B(n)$; a minor amount of backtracking at the start will not force a contradiction with the assumption that the filling of the 0-combing loop for $e$ satisfies the isodiametric condition. As a matter of fact, it is not even clear that the 1-combing described here will always be tame.

Theorem E. If a finitely presented group $G$ has a tame 1-combing admitting radial tameness function $\rho$ and ball tameness function $\beta$, then the function $f: \mathbf{N} \rightarrow \mathbf{N}$ given by $f(n)=n \cdot(\beta(\rho(n)))$ is an isoperimetric function, and the function $g: \mathbf{N} \rightarrow$ $\mathbf{N}$ given by $g(n)=J \cdot(\beta(\rho(n)))$ is an isodiametric function, for the group $G$.

Proof. Suppose that the universal cover of the standard 2-complex for a finite presentation of $G$ has a tame combing $\Psi$ admitting a radial tameness function $\rho$ and a ball tameness function $\beta$. Suppose also that $w$ is a freely reduced word representing the trivial element of $G$. The sea shell filling determines a van Kampen diagram for $w$.

For each edge $e$ in the boundary of the van Kampen diagram, the level of $e$ is at most the length $l(w)$, so all of the points in the combing paths from the identity to the points of $e$ must have level at most $\rho(l(w))$. The number of cells in $\Psi(e \times[0,1])$ with level in $[0, \rho(l(w))]$, counted with multiplicity, is bounded by $\beta(\rho(l(w)))$.

Since this is true for all of the edges in the boundary of the van Kampen diagram for $w$, the number of 2-cells in the diagram is at most $l(w) \cdot(\beta(\rho(l(w))))$. Therefore the function $f: \mathbf{N} \rightarrow \mathbf{N}$ given by $f(n)=n \cdot(\beta(\rho(n)))$ is an isoperimetric function for the group $G$. Also, any vertex inside the van Kampen diagram for $w$ is contained in the image of the homotopy for one of the edges, which also contains the basepoint and at most $\beta(\rho(l(w)))$ cells. Thus the vertex is at most a distance $J \cdot(\beta(\rho(l(w))))$ away from the basepoint of the diagram, where $J$ is the length of the longest relator of the presentation for $G$. Hence the function $g: \mathbf{N} \rightarrow \mathbf{N}$ given by $g(n)=$ $J \cdot(\beta(\rho(n)))$ is an isodiametric function for $G$.

Corollary 6.1. If a finitely presented group $G$ has a tame 1-combing admitting recursive radial and ball tameness functions, then $G$ has recursive isoperimetric and isodiametric functions, and the word problem for $G$ is solvable.

Proof. Since the composition of recursive functions is recursive 10, the isoperimetric and isodiametric functions in Theorem $\mathrm{E}$ are recursive functions. The rest follows the fact that a group with recursive isoperimetric function has solvable word problem (Theorem 2.2.5 of [3] or Theorem 2.1.2 of [5]). 


\section{REFERENCES}

[1] S.G. Brick, Quasi-isometries and amalgamations of tame combable groups, Internat. J. Comput. Algebra 5 (1995), 199-204. MR 96c:20064

[2] J.W. Cannon, Almost convex groups, Geom. Dedicata 22 (1987), 197-210. MR 88a:20049

[3] D.B.A. Epstein, J.W. Cannon, D.F. Holt, S.V.F. Levy, M.S. Paterson, and W.P. Thurston, Word Processing in Groups, Jones and Bartlett, Boston, 1992. MR 93i:20036

[4] S.M. Gersten, Dehn functions and $l_{1}$-norms of finite presentations, In: Algorithms and classification in combinatorial group theory, G. Baumslag, C.F. Miller III, eds., (M.S.R.I. Publications), Springer 23 (1992), 195-224. MR 94g:20049

[5] S.M. Gersten, Isoperimetric and isodiametric functions of finite presentations, In: Geometric group theory, vol. 1, G.A. Niblo and M.A. Roller, eds., (London Math. Soc. Lecture Note Ser.) Cambridge: Cambridge University Press 181 (1993), 79-96. MR 94f:20066

[6] S.M. Hermiller and J. Meier, Tame combings, almost convexity, and rewriting systems for groups, Math. Z. 225 (1997), 263-276. MR 98i:20036

[7] C. Hog-Angeloni, W. Metzler and A.J. Sieradski, eds., Two-dimensional Homotopy and Combinatorial Group Theory. London Math. Soc. Lecture Notes Series 197, Cambridge University Press, Cambridge, 1993. MR 95g:57006

[8] D.E. Knuth and P.B. Bendix, Simple word problems in universal algebras, In: Computational Problems in Abstract Algebra (Proc. Conf., Oxford, 1967) (1970), 263-297. MR 41:134

[9] R.C. Lyndon and P.E. Schupp, Combinatorial group theory, (Ergeb. Math., Bd. 89) Springer, Berlin-Heidelberg-New York, 1977. MR 58:28182

[10] M. Machtey and P. Young, An introduction to the general theory of algorithms, NorthHolland, New York, 1978. MR 81k:68001

[11] M.L. Mihalik, Compactifying coverings of 3-manifolds, Comment. Math. Helv. 71 (1996), 362-372. MR 97k:57020

[12] M.L. Mihalik, Group extensions and tame pairs, Trans. Amer. Math. Soc. 351 (1999), 10951107. MR 99e:57002

[13] M.L. Mihalik and S.T. Tschantz, Tame combings of groups, Trans. Amer. Math. Soc. 349 (1997), 4251-4264. MR 97m:20049

[14] V. Poénaru, Almost convex groups, Lipschitz combing, and $\pi_{1}^{\infty}$ for universal covering spaces of closed 3-manifolds, J. Differential Geom. 35 (1992), 103-130. MR 93d:57032

[15] V. Poénaru, Geometry "à la Gromov" for the fundamental group of a closed 3-manifold $M^{3}$ and the simple connectivity at infinity of $\widetilde{M}^{3}$, Topology 33 (1994), 181-196. MR 94m:57034

[16] V. Poénaru and C. Tanasi, k-weakly almost convex groups and $\pi_{1}^{\infty} \widetilde{M}^{3}$, Geom. Dedicata 48 (1993), 57-81. MR 94k:57003

[17] C.C. Sims, Computation with finitely presented groups. (Encyclopedia of Mathematics and its Applications 48), Cambridge University Press, Cambridge, 1994. MR 95f:20053

[18] C. Thiel, Zur Fast-Konvexität einiger nilpotenter Gruppen, Bonner Mathematische Schriften (1992). MR 95e:20052

[19] T. Tucker, Non-compact 3-manifolds and the missing boundary problem, Topology 13 (1974), 267-273. MR 50:5801

Department of Mathematics and Statistics, University of Nebraska, Lincoln, NeBRASKA 68588-0323

E-mail address: smh@math.unl.edu

Department of Mathematics, Lafayette College, Easton, Pennsylvania 18042

E-mail address: meierj@lafayette.edu 